\title{
Construction and test of the SM1 MicroMegas chambers for the upgrade of the forward Muon Spectrometer of the ATLAS experiment
}

\author{
G. Mancini on behalf of the ATLAS Muon Collaboration ${ }^{\mathrm{a}, *}$
}

${ }^{a}$ LNF INFN, Frascati, Italy

\begin{abstract}
MICRO MEsh GASeous structure chambers, MicroMegas (MM), is an innovative design concept for Micro-Pattern Gaseous Detectors, designed in order to provide a high spatial resolution and to cope with highly irradiated environments. These chambers have been chosen as new precision tracking detectors for the upgrade of the forward muon spectrometer of the ATLAS experiment. MM trapezoidal detector modules, with areas between 2 and $3 \mathrm{~m}^{2}$, will be part of the two New Small Wheels (NSW) composing the innermost station of the ATLAS muon tracking forward system. MM detectors are composed by 4 read-out layers, two for the reconstruction of the precision coordinate and two with stereo reading $\left( \pm 1.5^{\circ}\right)$ for the reconstruction of the second coordinate. MM chambers are designed to provide efficiency better than $95 \%$ per single plane at rate capability up to $15 \mathrm{kHz} / \mathrm{cm}^{2}$, in presence of magnetic fields up to 0.3 T. The Istituto Nazionale di Fisica Nucleare (INFN) is deeply involved in the construction of these chambers and in their validation at the Cosmic Ray Stand (CRS) in the Frascati INFN site (LNF). Preliminary results on the validation method and tests performed on INFN SM1 prototype at LNF are presented.
\end{abstract}

Keywords: Gas detectors, MicroMegas, Micro-pattern Gas Detectors

PACS: 29.40.Cs, 29.40.Gx

\section{Introduction and operating principles [1]}

Micromegas (MM) chambers operate in a gas mixture of Argon and Carbon Dioxide with a fraction of 93\% - 7\% respectively. The ionization by the charged particles in the detector gas produce $\sim 100$ pair $/ \mathrm{cm}$ considering both primary and secondary ionization. A Micromegas chamber is formed by the Read-Out structure (anode) based on a Printed Circuit Board (PCB), a fine micro-mesh, which defines the separation between the conversion and the amplification region, and the cathode. A schematic view of the working principle of a MM chamber is shown in Figure 1. The Read-Out PCBs consist of photo-lithographically etched copper strips, on top of which an additional layer done with resistive strips on a kapton foil is glued. Read-out and resistive strips are aligned and have both a pitch of $425-450 \mu \mathrm{m}$. The resistive strips have a resistivity of $\sim 10 \mathrm{M} \Omega / \mathrm{cm}$. The conversion drift region $\left(H V_{\text {drift }}=-300 \mathrm{~V}\right)$ and the amplification region $\left(H V_{\text {Read-Out }}=590 \mathrm{~V}\right)$ are asymmetric and respectively $5 \mathrm{~mm}$ and $128 \mu \mathrm{m}$ wide. Such a small amplification gap is guaranteed by the presence of insulating pillars with a diameter of $0.3 \mathrm{~mm}$ and a height of $128 \mu \mathrm{m}$ on top of the resistive layer. This structure guarantees a high transparency of the micromesh and a fast $(\sim 100 \mathrm{~ns})$ evacuation of positive ions produced in the avalanche through the micromesh. Two out of the four layers have strips parallel to the bases on the trapezoidal MM shape (aiming for the precision coordinate

\footnotetext{
${ }^{*}$ Corresponding author

Email address: giada.mancini@lnf .infn.it (G. Mancini on behalf of the ATLAS Muon Collaboration)
}

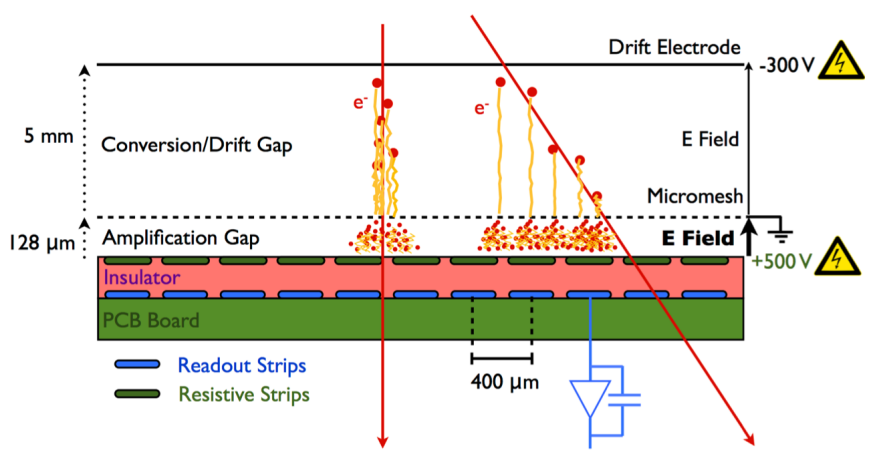

Figure 1: Schematic view of the working principle of a MM chamber.

reconstruction), while the others are inclined by $\pm 1.5^{\circ}$ for the $2^{\text {nd }}$ coordinate reconstruction.

\section{The New Small Wheel (NSW): MM for the ATLAS up- grade [2], [3]}

The NSW will replace the innermost station of the forward muon spectrometer in the end-cap region of the ATLAS detector. The upgrade will give an improvement with respect to the performances of the current Small Wheel (SW) in terms of trigger and tracking efficiency in view of the increasing luminosity for the next LHC Runs (up to $5-710^{34} \mathrm{~cm}^{-2} \mathrm{~s}^{-1}$ ). Two different detectors will be used for this purpose: Micromegas (aiming for precision tracking) and sTGCs (focused on the trigger). The NSW has a wheel-like structure and it is composed by 8 large sectors (LM) and 8 small ones (SM), therefore 2 MM modules 


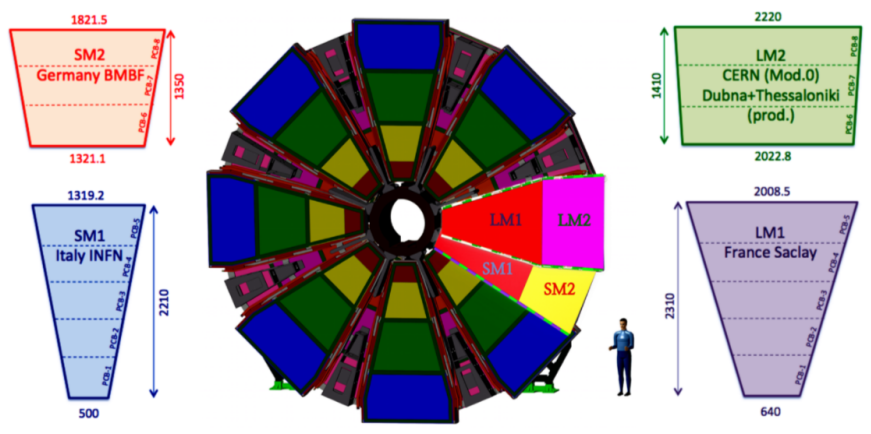

Figure 2: Wheel-like structure of the NSW highlighting the 4 MM modules (LM 1-2, SM 1-2) and the construction sites.
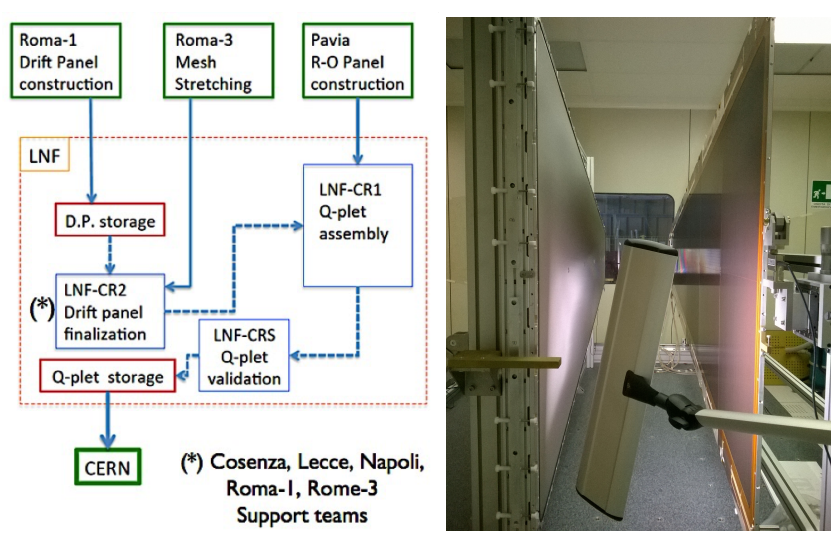

Figure 3: The SM1 production scheme is reported (left) together with a picture showing the vertical assembly (right).

per sector are needed which correspond to $4 \mathrm{MM}$ quadruplets per sector as shown in Figure 2. Four type of chambers are being constructed, namely LM 1-2, SM 1-2 whose production is distributed between several industries and institutes: Italy (SM1), Germany (SM2), France (LM1), Russia-Greece-CERN (LM2).

\section{SM1 production scheme}

The INFN group is responsible of the SM1 quadruplets construction. Mechanical precision measurements represent a challenge in the MM construction due to the strong constraints on the strip alignment on each RO layer: within $30 \mu \mathrm{m}$ RMS in $\eta$ (precision coordinate) and $80 \mu m$ RMS in $\mathrm{z}$ axis corresponding to the chamber planarity. As previously described, a single quadruplet is formed by 5 panels, 3 finalized drift panels ${ }^{1}$ ( 2 outer and a central) and 2 read out panels in order to define the quadruplet. Figure 3 shows the SM1 production scheme, together with a picture showing the vertical assembly.

\section{Preliminary results on first prototypes}

The read-out electronics used for the prototypes is based on APV25+SRS modules operating at $40 \mathrm{MHz}$ (final version

\footnotetext{
${ }^{1}$ A drift panel is finalized when the previously stretched micro-mesh is glued on the bare drift panel.
}
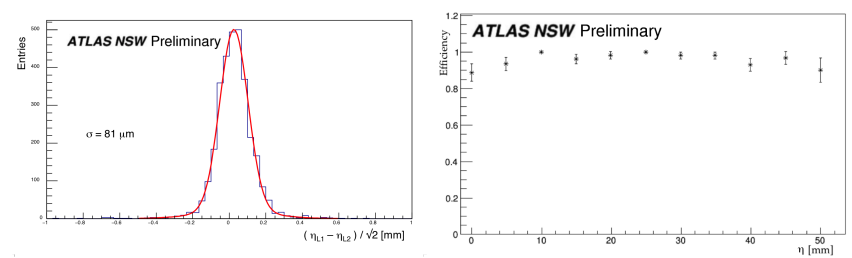

Figure 4: (left) Resolution on the precision coordinate of the Mod0 tested at the CERN H8 beamline. (right) Efficiency of the Mod0.5 tested at the LNF CRS.

forsee VMM ASICs). The APV25 are 128 channels chips whose signal corresponds to the values of the collected charge for different time samplings. For each event and each channel, a total of 18 samples (every $25 \mathrm{~ns}$ ), was recorded in an acquisition time window and then fitted with a Fermi-Dirac function (being the half-height parameter $t_{0}$ the signal start). Mod0 has been tested at the CERN H8 beam line of the SPS $\left(\pi^{+}\right.$beam at $180 \mathrm{GeV} / \mathrm{c}$ with a frequency between $1-500 \mathrm{kHz}$ and beam dimension of $1 \times 1 \mathrm{~cm}^{2}$ ) using $5 \mathrm{Tmm}$ chambers as external tracking chambers ${ }^{2}$, while Mod0.5 has been tested at the CRS of the LNF using 2 Tmm as reference. Pedestal has been subtracted and cross-talk effects between strips have been taken into account for the track hit reconstruction. The resolution on the precision coordinate is found to be $81 \mu \mathrm{m}$ and the efficiency $>95 \%$ from the measurements performed respectively on Mod0 (at CERN H8) and Mod0.5 (at LNF CRS). Results are well within the requirements as shown in Figure 4.

\section{Conclusions}

MM chambers will be used after Long Shutdown 2 (LS2) for the precision tracking in the NSWs of the ATLAS experiment. The INFN group has been the first to construct 2 full size prototypes (SM1 Mod0 and Mod0.5) and the performances of those quadruplets have been found to be within expectations in terms of efficiency and resolution for perpendicular tracks. HV instability effects emerged on the first built chambers and have been largely solved by defining a proper cleaning procedure of the chamber before assembly. The assembly and test procedures have been validated and we are ready for the production.

Copyright 2018 CERN for the benefit of the ATLAS Collaboration. CC-BY4.0 license.

\section{References}

[1] Y. Giomataris, P. Rebourgeard, J. Robert and G. Charpak, A High granularity position sensitive gaseous detector for high particle flux environments, Nucl. Instrum. Meth. A 376 (1996) 29.

[2] ATLAS collaboration, New Small Wheel, Technical Design Report, CERN-LHCC-2013-006 (2013).

[3] T. Alexopoulos et al., Development of large size Micromegas detector for the upgrade of the ATLAS muon system, Nucl. Instrum. Meth. A 617 (2010) 161

[4] T. Alexopoulos et al., A spark-resistant bulk-Micromegas chamber for high-rate applications, Nucl. Instrum. Meth. A 640 (2011) 110.

\footnotetext{
${ }^{2}$ Bulk-type MM with resistive strips $\left(10 \times 10 \mathrm{~cm}^{2}\right.$ of active area, $150 \mathrm{~mm}$ strip width and $250 \mathrm{~mm}$ strip pitch)[4].
} 